\title{
Surface-Wave Instabilities in a Plasma Rotating with Step-like Frequency Profile
}

\author{
A.B. Mikhailovskii and V.D. Pustovitov \\ Institute of Nuclear Fusion, Russian Research Centre Kurchatov Institute, 123182 Moscow, Russia \\ R.M.O. Galvão \\ Brazilian Center for Research in Physics, Rio de Janeiro, Brazil and \\ Institute of Physics, University of São Paulo, São Paulo, Brazil \\ C.H.S. Amador \\ Brazilian Center for Research in Physics, Rio de Janeiro, Brazil \\ J.G. Lominadze \\ Kharadze Abastumani National Astrophysical Observatory, 0160 Tbilisi, Georgia and \\ Nodia Institute of Geophysics, Tbilisi 0193 Tbilisi, Georgia \\ A.P. Churikov \\ Syzran Branch of Samara Technical University, 446001 Syzran, Samara Region, Russia
}

O.A. Kharshiladze

Nodia Institute of Geophysics, Tbilisi 0193 Tbilisi, Georgia

(Received on 26 November, 2008)

\begin{abstract}
An approach for the theory of nonlocal magnetorotational and convective instabilities in a rotating plasma is developed, on the basis of the surface wave concept, which allows the derivation of useful analytical results.It is assumed that the rotation frequency has a step-like profile, so that a narrow transition layer separates two regions with different rotation frequencies. The one-fluid magnetohydrodynamic (MHD) model for description of the perturbed plasma dynamics is used. It is shown that in the case of magnetized plasma the main properties of the nonlocal (surface-wave) instabilities are similar to those of the local ones.
\end{abstract}

Keywords: Magnetorotational instability; Plasma rotational instability

\section{INTRODUCTION AND OVERVIEW}

One of the important issues in recent plasma physics studies is the spontaneous generation of magnetic field in a rotating plasma, motivated by numerous investigations of this effect in a rotating liquid metals (see pioneering papers [1-3] and the review [4]).

Because most of the previous analytical works in this field, starting with Ref. 1, were based upon a local-mode analysis, it is important to complement them with other theoretical approaches that allow for a simple description of non-local modes. In particular, the surface waves model, based upon step-like profiles of the equilibrium quantities, has been recently applied in a simplified analysis of the magnetorotational instability (MRI) [5]. One of the goals of the present work is to develop a more general theory of nonlocal modes in the surface-wave approximation.

First, let us define the model of simplest astrophysical equilibrium. This is a cylindrical plasma rotating in azimuthal direction $\theta$ with the angular frequency $\Omega=\Omega(r)$, where $r$ is the radius. The equilibrium magnetic field $\mathbf{B}_{0}$ is assumed uniform and directed along the cylinder axis $z, \mathbf{B}_{0}=\left(0,0, B_{0}\right)$. The model also includes a gravitational force $\mathbf{g}$, with the radial component only, $\mathbf{g}=(g, 0,0)$, and an equilibrium electric field $\mathbf{E}_{0}=\left(E_{0}, 0,0\right)$ related to the rotation frequency $\Omega=V_{0} / r$ by $E_{0}=-r \Omega B_{0} / c$, where $V_{0}=V_{0}(r)$ is the azimuthal equilibrium plasma velocity and $c$ is the speed of light. The equilibrium plasma pressure $p_{0}$ is assumed uniform, $p_{0}^{\prime}=0$, where prime is the radial derivative. Then the radial equilibrium condition yields

$$
-r \Omega^{2}=g
$$

An alternative to the simplest astrophysical equilibrium is the simplest laboratory equilibrium. In this case $g=0$, while $p_{0}^{\prime} \neq 0$. Then, instead of (1.1), one has

$$
\rho_{0} r \Omega^{2}=p_{0}^{\prime},
$$

where $\rho_{0}$ is the equilibrium mass density.

A general case, when both $g$ and $p_{0}^{\prime}$ are finite, can be called the astrophysical equilibrium with pressure gradient. Then, instead of (1.1) and (1.2), one has

$$
\rho_{0} r \Omega^{2}=p_{0}^{\prime}-\rho_{0} g .
$$

The theory of nonlocal modes developed in Ref. 5 considered only the simplest astrophysical equilibrium, i.e., with $p_{0}^{\prime}=0$. In the present paper this theory is extended to equilibria with $p_{0}^{\prime} \neq 0$.

According to Ref. [1], with both $g \neq 0$ and $p_{0}^{\prime} \neq 0$ two varieties of instabilities may appear: the MRI and the convective instability, CI. Our analysis includes both of them.

An important subset of the theory of local instabilities in a rotating plasma is the modes with parallel phase velocities much smaller than the sound velocity in a high- $\beta$ plasma (the slow modes), where $\beta$ is the ratio of plasma pressure to the magnetic field pressure. Here, althoug a complete analytical technique for arbitrary modes and arbitrary $\beta$ is developed, we consider its applications primarily for slow modes in a high- $\beta$ plasma. 
One of the kee physics issues in this problem is the level of heat conductivity. In our analysis, preceded by Ref. [6] (see also Refs. $[7,8]$ ), both cases of high and finite heat conductivity are considered.

The analysis of nonlocal perturbations requires a proper mode equation. Its derivation is presented in Sec. 2, which summarizes the mathematical technique developed for the case of high heat conductivity in Refs. [9, 10] (see also Refs. [1114]) and for arbitrary heat conductivity in Refs. 15 and 16.

The main benchmarks of our technique are the pair of the canonical first-order differential equations for perturbations called the Hameiri-Bondeson-Iakono-Bhattacharjee (HBIB) type equations, first derived in $[12,13]$. The variables in these equations are the perturbed radial magnetic field $\widetilde{B}_{r}$, the socalled Friman-Rotenberg (FR) variable,

$$
p_{*}=\widetilde{p}+\widetilde{B}_{z} B_{0} /(4 \pi),
$$

and

$$
\tau_{B}=\frac{1}{r} \frac{\partial}{\partial r}\left(r \widetilde{B}_{r}\right)
$$

Here $\widetilde{p}$ is the perturbed plasma pressure and $\widetilde{B}_{z}$ is the parallel projection of the perturbed magnetic field. The mentioned canonical equations are

$$
\begin{gathered}
D \tau_{B}=C_{1} \widetilde{B}_{r}-i 4 \pi k_{z} B_{0} C_{2} p_{*}, \\
i 4 \pi k_{z} B_{0} D p_{*}^{\prime}=-4 \pi k_{z} B_{0} \bar{C}_{1} p_{*}+C_{3} \widetilde{B}_{r} .
\end{gathered}
$$

Here $D, C_{1}, \bar{C}_{1}, C_{2}$, and $C_{3}$ are the primary canonical parameters and $k_{z}$ is the parallel wave number. We call the effect related to $C_{1}$ the first oblique effect, while that described by $\bar{C}_{1}$ the second oblique effect. In $[9,10]$ it has been shown that the high heat conductivity leads to the following symmetry:

$$
\bar{C}_{1}=C_{1} .
$$

Below we prove that the same symmetry survives at arbitrary heat conductivity. This is important since in this case Eqs. (1.6) and (1.7) lead to a self-adjoint canonical mode equation

$$
D\left(D \tau_{B} / C_{2}\right)^{\prime}+\Lambda \widetilde{B}_{r}=0 .
$$

Here $\Lambda$ is the secondary canonical parameter,

$$
\Lambda=a+b
$$

with $a$ and $b$ the secondary local and differential canonical parameters, respectively, determined by

$$
\begin{gathered}
a=C_{3}-C_{1}^{2} / C_{2}, \\
b=-\operatorname{Dr}\left[C_{1} /\left(r C_{2}\right)\right]^{\prime} .
\end{gathered}
$$

In Sec. 3 we discuss the general aspects of the problem of slow modes in high- $\beta$ plasma. There we use the so-called quasi-incompressible approximation, when the "sound propagator"

$$
\alpha_{s}^{T}=1-\frac{p_{0} k_{z}^{2}\left(\Gamma \widetilde{\omega}+i \Delta_{T}\right)}{\rho_{0} \widetilde{\omega}^{2}\left(\widetilde{\omega}+i \Delta_{T}\right)}
$$

is replaced by

$$
\alpha_{s}^{T} \rightarrow-\frac{p_{0} k_{z}^{2}\left(\Gamma \widetilde{\omega}+i \Delta_{T}\right)}{\rho_{0} \widetilde{\omega}^{2}\left(\widetilde{\omega}+i \Delta_{T}\right)} .
$$

Here $\Gamma$ is the adiabatic exponent, $\Delta_{T}$ is the characteristic heatconductivity-induced decay rate, $\widetilde{\omega}=\omega-m \Omega$ is the Dopplershifted oscillation frequency with $\omega$ being the usual oscillation frequency and $m$ the azimuthal mode number. Then the symmetry expressed by Eq. (1.8), describing the equality of the first and second oblique effects, is automatically satisfied.

As an alternative to the quasi-incompressible approximation, there is the Boussinesq approximation. We explain in Sec. 3 that the latter leads to

$$
C_{1}=C_{1}^{\text {Bous }} \equiv 0,
$$

where the superscript "Bous" means Boussinesq. Then the first oblique effect disappears in the first canonical first-order differential equation, i.e., instead of (1.6), one has

$$
D \tau_{B}=-i 4 \pi k_{z} B_{0} C_{2} p_{*} .
$$

We show in Sec. 3 that, instead of Eq. (1.7), the Boussinesq approximation yields the second canonical first-order equation of the form

$$
i 4 \pi k_{z} B_{0} D p_{*}^{\prime}=C_{3} \widetilde{B}_{r}-i 4 \pi k_{z} B_{0} \bar{C}_{1}^{\text {Bous }} p_{*},
$$

where

$$
\bar{C}_{1}^{\text {Bous }}=\bar{C}_{1} .
$$

Thereby, in the Boussinesq approximation one deals with nonvanishing second oblique effect, $C_{1} \neq 0$. Excluding $p_{*}$ from Eq. (1.17) by means of Eq. (1.16), one arrives at the mode equation in the Boussinesq approximation

$$
D\left(D \tau_{B} / C_{2}\right)^{\prime}+\Lambda^{\text {Bous }} \widetilde{B}_{r}+\bar{C}_{1} D \tau_{B} / C_{2}=0,
$$

where

$$
\Lambda^{\text {Bous }}=C_{3} \text {. }
$$

Comparing Eqs. (1.19) and Eq. (1.9), one can see that the Boussinesq approximation leads to a non-self-adjoint mode equation with reduced expression for $\Lambda$. Using the Boussinesq approximation, Ref. 6 has neglected the term with $\bar{C}_{1}^{\text {Bous }}$ in Eq. (1.17). In this case the mode equation (1.19) is substituted by

$$
D\left(D \tau_{B} / C_{2}\right)^{\prime}+C_{3} \widetilde{B}_{r}=0 .
$$

Being central in the Balbus approximation this can be called the Balbus mode equation.

Section 4 is devoted to derivation of the nonlocal dispersion relation. In Sec. 5 the theory of nonlocal axisymmetric modes in the simplest astrophysical plasma is developed. In contrast, Sec. 6 considers the nonlocal axisymmetric modes allowing for both the gravitation force and the plasma pressure gradient. In Sec. 7 we transit to analysis of the nonaxisymmetric modes. Discussion of the results is given in Sec. 8.

It seems reasonable to compare the results of nonlocal and local theories. Our paper contains Appendices summarizing the local results obtained in [9, 15]: Appendix A shows the general preliminary results, while Appendices $\mathrm{B}$ and $\mathrm{C}$ are related to the axisymmetric and nonaxisymmetric modes, respectively. 


\section{BASIC EQUATIONS AND GENERAL TRANSFORMATIONS}

\subsection{Basic equations}

We start with the standard MHD plasma motion equation

$$
\rho \frac{d \mathbf{V}}{d t}=-\nabla p+\rho \mathbf{g}-\frac{1}{4 \pi}\left\{\nabla \frac{\mathbf{B}^{2}}{2}-(\mathbf{B} \cdot \nabla) \mathbf{B}\right\},
$$

where $\mathbf{V}$ is the plasma velocity, $\mathbf{B}$ the magnetic field, $p$ the plasma pressure, $\rho$ is the plasma mass density, $\mathbf{g}$ is the gravitation force, and $d / d t=\partial / \partial t+\mathbf{V} \cdot \nabla$. We use the Ohm law in the form $\mathbf{E}+[\mathbf{V} \times \mathbf{B}] / c=0$, where $\mathbf{E}$ is the electric field and $c$ is the speed of light. This equation leads to the standard frozen-in condition

$$
\partial \mathbf{B} / \partial t-\nabla \times[\mathbf{V} \times \mathbf{B}]=0 .
$$

In addition, we use the Maxwell equation

$$
\nabla \cdot \mathbf{B}=0
$$

the plasma continuity equation

$$
d \rho / d t+\rho \nabla \cdot \mathbf{V}=0,
$$

and the heat conductivity equation

$$
n \frac{d T}{d t}+(\Gamma-1) p \nabla \cdot \mathbf{V}=-(\Gamma-1) \nabla \cdot \mathbf{q}
$$

Here $\Gamma=5 / 3$ is the adiabatic exponent, $n=\rho / M$ is the plasma number density, $M$ is the ion mass, $T$ is the plasma temperature related to the pressure and density by $p=n T, \mathbf{q}$ is the heat flux defined by

$$
\mathbf{q}=-\kappa_{T} \mathbf{b}(\mathbf{b} \cdot \nabla T)
$$

where $\kappa_{T}$ is the heat conductivity coefficient, $\mathbf{b}=\mathbf{B} / B$.

We consider a cylindrical plasma rotating in azimuthal direction $\theta$ with the angular frequency $\Omega=\Omega(r)$ ( $r$ is the radius), which was described in Sec. 1 as the astrophysical equilibrium with pressure gradient, see Eq. (1.3). We linearize the basic equations assuming each perturbation to depend on $t, \theta$, $z$ as $\exp \left(-i \omega t+i m \theta+i k_{z} z\right)$, where $\omega$ is the oscillation frequency, $m$ is the azimuthal mode number, $k_{z}$ is the parallel projection of the wave vector. In addition to $m$, we introduce $k_{y} \equiv m / r$, the azimuthal projection of the wave vector, while, in addition to $\omega$, we use the Doppler-shifted oscillation frequency $\widetilde{\omega}=\omega-m \Omega$. The $(r, \theta, z)$-th projections of the perturbed plasma velocity $\widetilde{\mathbf{V}}$ are $\widetilde{V}_{r}, \widetilde{V}_{\theta}$, and $\widetilde{V}_{z}$. Similarly, the $(r, \theta, z)$ components of the perturbed magnetic field $\widetilde{\mathbf{B}}$ are $\widetilde{B}_{r}$, $\widetilde{B}_{\theta}$, and $\widetilde{B}_{z}$. The perturbed plasma mass density is designated by $\widetilde{\rho}$.
The $(r, \theta)$-th projections of the frozen-in condition (2.2) yield

$$
\begin{array}{r}
-i \widetilde{\omega} \widetilde{B}_{r}-i k_{z} B_{0} \widetilde{V}_{r}=0, \\
-i \widetilde{\omega} \widetilde{B}_{\theta}-\frac{d \Omega}{d \ln r} \widetilde{B}_{r}-i k_{z} B_{0} \widetilde{V}_{\theta}=0 .
\end{array}
$$

The Maxwell equation (2.3) gives

$$
i k_{z} \widetilde{B}_{z}+i k_{y} \widetilde{B}_{\theta}+\frac{1}{r} \frac{\partial}{\partial r}\left(r \widetilde{B}_{r}\right)=0 .
$$

The perturbed plasma motion equation (2.1) yields

$$
-i \widetilde{\omega} \widetilde{V}_{r}-2 \Omega \widetilde{V}_{\theta}+\frac{1}{\rho_{0}} \frac{\partial \widetilde{p}}{\partial r}-\frac{i v_{A}^{2} k_{z}}{B_{0}} \widetilde{B}_{r}+\frac{v_{A}^{2}}{B_{0}} \frac{\partial \widetilde{B}_{z}}{\partial r}-\frac{\widetilde{\rho} p_{0}^{\prime}}{\rho_{0}^{2}}=0,
$$

$$
-i \widetilde{\omega} \widetilde{V}_{\theta}+\frac{\kappa^{2}}{2 \Omega} \widetilde{V}_{r}+\frac{i k_{y} \widetilde{p}}{\rho_{0}}-\frac{i v_{A}^{2} k_{z}}{B_{0}} \widetilde{B}_{\theta}+\frac{i v_{A}^{2}}{B_{0}} k_{y} \widetilde{B}_{z}=0
$$

$$
-i \widetilde{\omega} \widetilde{V}_{z}=-i k_{z} \widetilde{p} / \rho_{0}
$$

Here $v_{A}^{2}=B_{0}^{2} /\left(4 \pi \rho_{0}\right)$ is the Alfvén velocity squared, $\kappa^{2}=$ $(2 \Omega / r) d\left(r^{2} \Omega\right) / d r$, and the gravitation force is replaced through $\Omega$ and $p_{0}^{\prime}$ by means of Eq. (1.3).

With Eq. (2.9) we express $\widetilde{B}_{z}$ in terms of $\widetilde{B}_{r}, \widetilde{B}_{\theta}$ :

$$
\widetilde{B}_{z}=-\frac{k_{y}}{k_{z}} \widetilde{B}_{\theta}+\frac{i}{k_{z}} \tau_{B}
$$

where $\tau_{B}$ is given by Eq. (1.5).

With the FR variable $p_{*}$ defined by Eq. (1.4) Eqs. (2.10) and (2.11) take the form

$$
\begin{array}{r}
i\left(D_{0}-\frac{d \Omega^{2}}{d \ln r}\right) \widetilde{B}_{r}+2 \Omega \widetilde{\omega} \widetilde{B}_{\theta}+\frac{k_{z} B_{0}}{\rho_{0}} \frac{d p_{*}}{d r}-\frac{k_{z} B_{0} \widetilde{\rho} p_{0}^{\prime}}{\rho_{0}^{2}}=0, \\
i D_{0} \widetilde{B}_{\theta}-2 \Omega \widetilde{\omega} \widetilde{B}_{r}+\frac{i k_{y} k_{z} B_{0}}{\rho_{0}} p_{*}=0,
\end{array}
$$

where $D_{0}=\alpha_{A} \widetilde{\omega}^{2}, \alpha_{A}=1-k_{z}^{2} v_{A}^{2} / \widetilde{\omega}^{2}$.

It follows from Eq. (2.4), (2.7) and (2.9) that the perturbed density satisfies the equation

$$
\frac{\widetilde{\rho}}{\rho_{0}}=\frac{1}{k_{z} B_{0}}\left[i\left(\tau_{B}+\frac{d \ln \rho_{0}}{d r} \widetilde{B}_{r}\right)-k_{y} \widetilde{B}_{\theta}\right]+\frac{k_{z} \widetilde{V}_{z}}{\widetilde{\omega}} .
$$

Now we linearize Eq. (2.5) obtaining [cf. (2.16)]

$$
\frac{\widetilde{T}}{T_{0}}=\frac{1}{k_{z} B_{0}}\left[\frac{\widetilde{\omega}}{\left(\widetilde{\omega}+i \Delta_{T}\right)}(\Gamma-1)\left(i \tau_{B}-k_{y} \widetilde{B}_{\theta}+\frac{k_{z}^{2} B_{0}}{\widetilde{\omega}} \widetilde{V}_{z}\right)+\frac{i d \ln T_{0}}{d r} \widetilde{B}_{r}\right]
$$


Here $\Delta_{T}$ is the characteristic heat-conductivity-induced decay rate given by $\Delta_{T}=\kappa_{T}(\Gamma-1) k_{z}^{2} / n_{0}$. At last, we have

$$
\widetilde{p}=\left(\widetilde{\rho} / \rho_{0}+\widetilde{T} / T_{0}\right) p_{0}
$$

\subsection{Derivation of general canonical mode equation}

\subsubsection{Pair of canonical first-order differential equations}

Substituting Eqs. (2.16) and (2.17) into Eq. (2.18), one has $\widetilde{p}=\frac{p_{0}}{k_{z} B_{0}}\left[\left(i \tau_{B}-k_{y} \widetilde{B}_{\theta}+\frac{k_{z}^{2} \widetilde{V}_{z} B_{0}}{\widetilde{\omega}}\right) \frac{\Gamma \widetilde{\omega}+i \Delta_{T}}{\widetilde{\omega}+i \Delta_{T}}+i \frac{d \ln \rho_{0}}{d r} \widetilde{B}_{r}\right]$.

It follows from Eqs. (2.12) and (2.19) that

$$
\widetilde{V}_{z}=\frac{p_{0}}{\rho_{0} B_{0} \widetilde{\omega} \alpha_{s}^{T}}\left[\left(i \tau_{B}-k_{y} \widetilde{B}_{\theta}\right) \frac{\Gamma \widetilde{\omega}+i \Delta_{T}}{\widetilde{\omega}+i \Delta_{T}}+i \frac{d \ln \rho_{0}}{d r} \widetilde{B}_{r}\right] .
$$

Here $\alpha_{s}^{T}$ is the "sound propagator" defined by Eq. (1.13). Substitution of Eq. (2.20) into Eqs. (2.19) and (2.16) yields

$$
\begin{gathered}
\tilde{p}=\frac{p_{0}}{k_{z} B_{0} \alpha_{s}^{T}}\left[\frac{\Gamma \widetilde{\omega}+i \Delta_{T}}{\widetilde{\omega}+i \Delta_{T}}\left(i \tau_{B}-k_{y} \widetilde{B}_{\theta}\right)+i \frac{d \ln p_{0}}{d r} \widetilde{B}_{r}\right], \\
\frac{\widetilde{\rho}}{\rho_{0}}=\frac{1}{\alpha_{s}^{T} k_{z} B_{0}}\left[i \tau_{B}-k_{y} \widetilde{B}_{\theta}+i\left(\alpha_{s}^{T} \frac{d \ln \rho_{0}}{d r}+\frac{k_{z}^{2} p_{0}}{\rho_{0} \widetilde{\omega}^{2}} \frac{d \ln p_{0}}{d r}\right) \widetilde{B}_{r}\right] .
\end{gathered}
$$

Substituting Eq. (1.4) into Eq. (2.14), we arrive at

$$
i \lambda_{r} \widetilde{B}_{r}+\lambda_{\theta} \widetilde{B}_{\theta}-i \lambda_{\tau} \tau_{B}+k_{z} B_{0} p_{*}^{\prime} / \rho_{0}=0
$$

Here

$$
\begin{aligned}
& \lambda_{r}=D_{0}-\frac{d \Omega^{2}}{d \ln r}-\frac{p_{0}^{\prime}}{\rho_{0} \alpha_{s}^{T}}\left(\alpha_{s}^{T} \frac{d \ln \rho_{0}}{d \ln r}+\frac{k_{z}^{2} p_{0}}{\rho_{0} \widetilde{\omega}^{2}} \frac{d \ln p_{0}}{d r}\right), \\
& \lambda_{\theta}=2 \Omega \widetilde{\omega}+k_{y} p_{0}^{\prime} /\left(\rho_{0} \alpha_{s}^{T}\right) \\
& \lambda_{\tau}=p_{0}^{\prime} /\left(\rho_{0} \alpha_{s}^{T}\right) .
\end{aligned}
$$

It follows from Eq. (2.15) that

$$
\widetilde{B}_{\theta}=-\frac{1}{D_{0}}\left(i 2 \Omega \widetilde{\omega} \widetilde{B}_{r}+\frac{k_{y} k_{z} B_{0}}{\rho_{0}} p_{*}\right) .
$$

Substitution of Eq. (2.27) into Eqs. (2.13) and (2.21) leads to

$$
\begin{aligned}
\widetilde{B}_{z}= & \frac{i}{k_{z}}\left(\tau_{B}+\frac{k_{y}}{D_{0}} 2 \Omega \widetilde{\omega} \widetilde{B}_{r}\right)+\frac{k_{y}^{2}}{\rho_{0} p_{0}} B_{0} p_{*}, \\
\widetilde{p}= & \frac{p_{0}}{k_{z} B_{0} \alpha_{s}^{T}}\left\{i \frac{\Gamma \widetilde{\omega}+i \Delta_{T}}{\widetilde{\omega}+i \Delta_{T}} \tau_{B}+i\left[\frac{d \ln p_{0}}{d r}+\right.\right. \\
& \left.\left.+\frac{2 \Omega \widetilde{\omega} k_{y}\left(\Gamma \widetilde{\omega}+i \Delta_{T}\right)}{\left(\widetilde{\omega}+i \Delta_{T}\right) D_{0}}\right] \widetilde{B}_{r}+\frac{\Gamma \widetilde{\omega}+i \Delta_{T}}{\left(\widetilde{\omega}+i \Delta_{T}\right) D_{0}} \frac{k_{y}^{2} k_{z} B_{0}}{\rho_{0}} p_{*}\right\} .
\end{aligned}
$$

With Eqs. (2.28) and (2.29), Eq. (1.4) is represented in the form (1.6) where

$D=D_{0}\left(1+\frac{4 \pi p_{0}}{B_{0}^{2} \alpha_{S}^{T}} \frac{\Gamma \widetilde{\omega}+i \Delta_{T}}{\widetilde{\omega}+i \Delta_{T}}\right)$

$C_{1}=-\left[2 \Omega \widetilde{\omega} k_{y}\left(1+\frac{\Gamma \widetilde{\omega}+i \Delta_{T}}{\widetilde{\omega}+i \Delta_{T}} \frac{4 \pi p_{0}}{B_{0}^{2} \alpha_{s}^{T}}\right)+\frac{4 \pi p_{0} D_{0}}{B_{0}^{2} \alpha_{s}^{T}} \frac{d \ln p_{0}}{d r}\right]$,

$C_{2}=\frac{1}{B_{0}^{2}}\left\{D_{0}-k_{y}^{2} v_{A}^{2}\left[1+\frac{\Gamma \widetilde{\omega}+i \Delta_{T}}{\widetilde{\omega}+i \Delta_{T}} \frac{4 \pi p_{0}}{B_{0}^{2} \alpha_{s}^{T}}\right]\right\}$.

Equation (1.6) is the first canonical first-order differential equation of the MHD approach. The effect described by the coefficient $C_{1}$ is the first oblique effect. It can be seen that this effect is revealed only if $p_{0}^{\prime} \neq 0$ or $k_{y} \neq 0$.

Substitution of Eq. (2.27) into Eq. (2.23) yields

$$
-i p_{*}^{\prime}=-\frac{i k_{y} \lambda_{\theta}}{D_{0}} p_{*}+\frac{\rho_{0} \lambda_{\tau}}{k_{z} B_{0}} \tau_{B}-\frac{\rho_{0}}{k_{z} B_{0}}\left(\lambda_{r}-\frac{\lambda_{\theta}}{D_{0}} 2 \Omega \widetilde{\omega}\right) \widetilde{B}_{r} .
$$

Using Eq. (1.6), we exclude $\tau_{B}$ from Eq. (2.33). Then we arrive at Eq. (1.7) where

$$
\begin{aligned}
& \bar{C}_{1}=-\left[4 \pi \rho_{0} \lambda_{\tau} C_{2}+\lambda_{\theta} k_{y}\left(1+\frac{\Gamma \widetilde{\omega}+i \Delta_{T}}{\widetilde{\omega}+i \Delta_{T}} \frac{4 \pi p_{0}}{B_{0}^{2} \alpha_{s}^{T}}\right)\right], \\
& C_{3}=4 \pi \rho_{0}\left\{D\left(\lambda_{r}-\frac{\lambda_{\theta}}{D_{0}} 2 \Omega \widetilde{\omega}\right)-\lambda_{\tau} C_{1}\right\} .
\end{aligned}
$$

By means of Eqs. (2.31) and (2.32), Eqs. (2.34) and (2.35) reduce to Eq. (1.8) and

$$
\begin{aligned}
C_{3} & =4 \pi \rho_{0} D_{0}\left[\left(1+\frac{\Gamma \widetilde{\omega}+i \Delta_{T}}{\widetilde{\omega}+i \Delta_{T}} \frac{4 \pi p_{0}}{B_{0}^{2} \alpha_{s}^{T}}\right) \times\right. \\
& \times\left(D_{0}-\frac{d \Omega^{2}}{d \ln r}-\frac{p_{0}^{\prime}}{\rho_{0}} \frac{d \ln \rho_{0}}{d r}-\right. \\
& \left.\left.-\frac{4 \Omega^{2} \widetilde{\omega}^{2}}{D_{0}}\right)+\frac{D_{0} p_{0}^{\prime} p_{0}}{\rho_{0}^{2} v_{A}^{2} \alpha_{s}^{T} \widetilde{\omega}^{2}} \frac{d \ln p_{0}}{d r}\right] .
\end{aligned}
$$

Equation (1.7) is the second canonical first-order differential equation of the MHD approach. As a whole, Eqs. (1.7) and (1.8) are the Hameiri-Bondeson-Iakono-Bhattacharjee (HBIB) type equations. The effect described by the coefficient $\bar{C}_{1}$ is the second oblique effect.

\subsubsection{General canonical mode equation}

In order to exclude the value $p_{*}$ from our problem, we find from Eq. (1.7)

$$
i 4 \pi k_{z} B_{0} p_{*}=\left(C_{1} \widetilde{B}_{r}-D \tau_{B}\right) / C_{2} .
$$

Then equation (1.8) takes the form of Eq. (1.9) with $\Lambda$ given by Eqs. (1.10)-(1.12). 
3. MODE EQUATIONS IN THE QUASI-INCOMPRESSIBLE, BOUSSINESQ, AND BALBUS APPROXIMATIONS

\subsection{Quasi-incompressible approximation}

We introduce the quasi-incompressible approximation assuming the parameter $k_{z}^{2} p_{0}\left(\Gamma \widetilde{\omega}+i \Delta_{T}\right) /\left[\rho_{0} \widetilde{\omega}^{2}\left(\widetilde{\omega}+i \Delta_{T}\right)\right]$ to be large but finite. Then the sound propagator given by Eq. (1.13) reduces to (1.14), which is relevant to the high- $\beta$ plasma and the slow modes with parallel phase velocities much smaller than the sound velocity.

With Eq. (1.14), Eqs. (2.30)-(2.32), and (2.36) are transformed into

$$
D=-D_{0}^{2} /\left(k_{z}^{2} v_{A}^{2}\right) .
$$

$C_{1}=-\frac{D_{0}}{k_{z}^{2} v_{A}^{2}}\left[2 \Omega \widetilde{\omega} k_{y}+\widetilde{\omega}^{2} \frac{p_{0}^{\prime}}{\rho_{0}}\left(\frac{\widetilde{\omega}+i \Delta_{T}}{\Gamma \widetilde{\omega}+i \Delta_{T}} \frac{d \ln p_{0}}{d r}-\frac{d \ln \rho_{0}}{d r}\right)\right]$,

$$
C_{2}=\frac{1}{B_{0}^{2}} D_{0}\left(1+\frac{k_{y}^{2}}{k_{z}^{2}}\right),
$$

By means of the above expressions for $C_{1}, C_{2}$, and $C_{3}$, Eq. (1.11) reduces to

$$
\begin{aligned}
a= & -\frac{4 \pi \rho_{0} D_{0}^{2}}{k_{z}^{2} v_{A}^{2}}\left[D_{0}-\frac{d \Omega^{2}}{d \ln r}+\frac{p_{0}^{\prime}}{\rho_{0}}\left(\frac{\widetilde{\omega}+i \Delta_{T}}{\Gamma \widetilde{\omega}+i \Delta_{T}} \frac{d \ln p_{0}}{d r}-\frac{d \ln \rho_{0}}{d r}\right)-\right. \\
& \left.-\frac{d \ln \rho_{0}}{d r} \frac{4 \Omega^{2} \widetilde{\omega}^{2} k_{z}^{2}}{\left(k_{z}^{2}+k_{y}^{2}\right) D_{0}}+a_{3}+a_{4}\right],
\end{aligned}
$$

where

$$
\begin{aligned}
& a_{3}=\frac{4 k_{y} \Omega \widetilde{\omega}^{3}\left(\widetilde{\omega}+i \Delta_{T}\right)}{\left(k_{y}^{2}+k_{z}^{2}\right)\left(\Gamma \widetilde{\omega}+i \Delta_{T}\right) D_{0}} \frac{d \ln p_{0}}{d r} \\
& a_{4}=\frac{\widetilde{\omega}^{4}\left(\widetilde{\omega}+i \Delta_{T}\right)^{2}}{\left(k_{y}^{2}+k_{z}^{2}\right)\left(\Gamma \widetilde{\omega}+i \Delta_{T}\right)^{2} D_{0}}\left(\frac{d \ln p_{0}}{d r}\right)^{2} .
\end{aligned}
$$

Similarly, Eq. (1.12) is transformed to

$$
\begin{aligned}
b & =\frac{4 \pi D_{0}^{2}}{v_{A}^{2}} \frac{d}{d \ln r}\left\{\frac { \rho _ { 0 } } { r ( k _ { z } ^ { 2 } + k _ { y } ^ { 2 } ) } \left[2 \Omega \widetilde{\omega} k_{y}+\right.\right. \\
& \left.\left.+\frac{\widetilde{\omega}^{2}\left(\widetilde{\omega}+i \Delta_{T}\right)}{\left(\Gamma \widetilde{\omega}+i \Delta_{T}\right)} \frac{d \ln p_{0}}{d r}\right]\right\} .
\end{aligned}
$$

\subsection{The Boussinesq approximation}

By means of Eq. (2.4), one can exclude $\nabla \cdot \mathbf{V}$ from Eq. (2.5) arriving at the entropy equation

$$
p \frac{d}{d t} \ln \left(\frac{p}{\rho^{\Gamma}}\right)=-(\Gamma-1) \nabla \cdot \mathbf{q} .
$$

Neglecting the term $d \rho / d t$ in Eq. (2.4), one has the incompressibility condition

$$
\nabla \cdot \mathbf{V}=0 .
$$

Using Eq. (3.10), instead of Eq. (2.4), together with Eq. (3.9), is the essence of the Boussinesq approximation.

In the case of axisymmetric perturbations Eq. (3.10) yields

$$
\frac{1}{r} \frac{\partial}{\partial r}\left(r \widetilde{V}_{r}\right)+i k_{z} \widetilde{V}_{z}=0
$$

Hence, using Eq. (2.7), one has

$$
\widetilde{V}_{z}=-\frac{i \omega}{k_{z}^{2} B_{0}} \tau_{B}
$$

By means of Eq. (2.12) and (3.12), we arrive at

$$
\widetilde{p}=-\frac{i \omega^{2} \rho_{0}}{k_{z}^{3} B_{0}} \tau_{B}
$$

With Eq. (2.13) for $k_{y}=0$ and (3.13), Eq. (1.4) leads to

$$
p_{*}=\frac{i B_{0}}{4 \pi k_{z}}\left(1-\frac{\omega^{2}}{k_{z}^{2} v_{A}^{2}}\right) \tau_{B}
$$

This is the same as Eq. (1.6) for $D$ and $C_{2}$ given by Eqs. (3.1) and (3.3) (for $k_{y}=0$ ) and $C_{1}=0$, (see Eq. (1.15)).

Turning to Eq. (3.9), one finds 


$$
\frac{\widetilde{\rho}}{\rho_{0}}=\frac{1}{\Gamma \omega+i \Delta_{T}}\left\{\left(\omega+i \Delta_{T}\right) \frac{\widetilde{p}}{p_{0}}-i\left[\omega \frac{d}{d r} \ln \left(\frac{p_{0}}{\rho_{0}^{\Gamma}}\right)+i \Delta_{T} \frac{d \ln T_{0}}{d r}\right] \frac{\widetilde{B}_{r}}{k_{z} B_{0}}\right\} .
$$

Substitution of Eq. (3.13) into (3.15) yields

$\frac{\widetilde{\rho}}{\rho_{0}}=\frac{-i}{k_{z} B_{0}}\left[\frac{\omega+i \Delta_{T}}{\Gamma \omega+i \Delta_{T}}\left(\frac{i \omega^{2} \rho_{0}}{k_{z} p_{0}} \tau_{B}+\frac{d \ln \rho_{0}}{d r} \widetilde{B}_{r}\right)-\frac{d \ln \rho_{0}}{d r} \widetilde{B}_{r}\right]$.

It follows from Eq. (2.15) for $k_{y}=0$ that

$$
\widetilde{B}_{\theta}=-i 2 \Omega \omega \widetilde{B}_{r} / D_{0}
$$

Substitution of Eq. (3.17) into Eq. (2.14) leads to

$$
i\left(D_{0}-\frac{d \Omega^{2}}{d \ln r}-\frac{4 \Omega^{2} \omega^{2}}{D_{0}}\right) \widetilde{B}_{r}+\frac{k_{z} B_{0}}{\rho_{0}} \frac{d p_{*}}{d r}-\frac{k_{z} B_{0} \widetilde{\rho} p_{0}^{\prime}}{\rho_{0}^{2}}=0 .
$$

For $\widetilde{\rho}$ given by Eq. (3.16) this equation means

$$
i 4 \pi k_{z} B_{0} D p_{*}^{\prime}=C_{3} \widetilde{B}_{r}+i 4 \pi D p_{0}^{\prime} \frac{\omega+i \Delta_{T}}{\Gamma \omega+i \Delta_{T}} \frac{\omega^{2} \rho_{0}}{k_{z}^{2} p_{0}} \tau_{B},
$$

where the constants $D$ and $C_{3}$ are defined by Eqs. (3.1) and (3.4), respectively (for $k_{y}=0$ ). Using Eq. (3.13), one can express $\tau_{B}$ in terms of $p_{*}$ and arrive at an equation similar to Eq. (1.7), which is given by Eq. (1.17), where

$$
\bar{C}_{1}^{\text {Bous }}=\frac{\omega^{2} D_{0}}{k_{z}^{2} v_{A}^{2}} \frac{p_{0}^{\prime}}{p_{0}} \frac{\omega+i \Delta_{T}}{\Gamma \omega+i \Delta_{T}} .
$$

Comparing Eq. (3.20) with Eq. (2.34) for $k_{y}=0$ and using (1.14), one has (1.18). Then one arrives at the mode equation in the Boussinesq approximation given by (1.19), (1.20).

\subsection{The Balbus approximation}

In Ref. 6 , the term with $\widetilde{p}$ in Eq. (3.15) for $\widetilde{\rho}$ has been neglected. This followed by the statement that such a neglect "is an implementation of the Boussinesq approximation (relative changes in the pressure are much smaller than relative changes in the temperature or density)". Then one arrives at the Balbus mode equation (1.21).

\section{DERIVATION OF NONLOCAL DISPERSION RELATION}

We represent (1.9) as

$$
\frac{\partial}{\partial r}\left[G \frac{1}{r} \frac{\partial}{\partial r}\left(r \widetilde{B}_{r}\right)\right]-H \widetilde{B}_{r}=0
$$

where

$$
\begin{aligned}
& G=D / C_{2}, \\
& H=-\Lambda / D .
\end{aligned}
$$

In the quasi-slab approximation, (4.1) reduces to

$$
\frac{\partial}{\partial r}\left(G \frac{\partial \widetilde{B}_{r}}{\partial r}\right)-H \widetilde{B}_{r}=0 .
$$

Assume that there is a jump of the rotation frequency $\Omega$ at $r=r_{0}$ from $\Omega_{1}$ to $\Omega_{2}$, where " 1 " means $r<r_{0}$ (the inside region) while " 2 " denotes $r>r_{0}$ (the outside region). The characteristic width of the transition layer between the regions is $a \ll r_{0}$.

Far from the transition layer the functions $G$ and $H$ can by approximated by constants. Then solution of Eq. (4.4) decreasing with increasing $\left|r-r_{0}\right|$ is given by

$$
\left(\widetilde{B}_{r}\right)_{1,2}=\widetilde{B}_{r}^{(0)} \exp \left(-\sigma_{1,2}\left|r-r_{0}\right|\right)
$$

where $\widetilde{B}_{r}^{(0)}$ is a constant and

$$
\sigma_{1,2}=\left(H_{1,2} / G_{1,2}\right)^{1 / 2}
$$

Now we integrate Eq. (4.4) over a region of the width of order $\delta$ including the transition layer assuming $a \ll \delta \ll 1 / \sigma_{1,2}$ and $\widetilde{B}_{r}$ to be continuous. Then we obtain

$$
\left.\frac{D}{C_{2}}\left(\frac{\partial \widetilde{B}_{r}}{\partial r}\right)\right|_{r_{0}-\delta} ^{r_{0}+\delta}-\widetilde{B}_{r}^{(0)} I=0
$$

where

$$
I=\int_{r_{0}-\delta}^{r_{0}+\delta} H d r
$$

Substitution of Eq. (4.5) into (4.7) leads to

$$
(G H)_{2}^{1 / 2}+(G H)_{1}^{1 / 2}+I=0 .
$$

This is the nonlocal dispersion relation.

In terms of the canonical parameters one has

$$
(G H)_{2,1}^{1 / 2}=\left(-\Lambda / C_{2}\right)_{2,1}^{1 / 2}
$$

where

$$
I=-\int_{r_{0}-\delta}^{r_{0}+\delta} d r \Lambda / D
$$

The parameters $C_{1}$ and $C_{2}$ do not contain the $\delta$-functional singularity. Then (4.11) reduces to

$$
I=-\int_{r_{0}-\delta}^{r_{0}+\delta} \frac{C_{3}}{D} d r+\left.\left(\frac{C_{1}}{C_{2}}\right)\right|_{1} ^{2}
$$


On the other hand, (4.10) is transformed to

$$
(G H)_{2,1}^{1 / 2}=\left[\left(C_{1}^{2}-C_{2} C_{3}\right) / C_{2}^{2}\right]_{2,1}^{1 / 2} .
$$

The term $I$ describes the nonlocal part of the nonlocal dispersion relation (4.9), while $(G H)_{2,1}^{1 / 2}$ the local one.

According to Eqs. (3.1)-(3.4), in the case $m=0\left(k_{y}=0\right)$, $p_{0}^{\prime}=0, \rho_{0}^{\prime}=0, \beta \gg 1, \Delta_{T} \rightarrow 0$, i.e., in the case of slow axisymmetric modes in the simplest astrophysical plasma for low heat conductivity, one has

$$
\begin{aligned}
D & =-\left(\omega^{2}-k_{z}^{2} v_{A}^{2}\right)^{2} / k_{z}^{2} v_{A}^{2}, \\
C_{1} & =0, \\
C_{2} & =\left(\omega^{2}-k_{z}^{2} v_{A}^{2}\right) / B_{0}^{2}, \\
C_{3} & =-4 \pi \rho_{0}\left(\omega^{2}-k_{z}^{2} v_{A}^{2}\right)^{2}\left(\omega^{2}-k_{z}^{2} v_{A}^{2}-\frac{d \Omega^{2}}{d \ln r}-\frac{4 \Omega^{2} \omega^{2}}{\omega^{2}-k_{z}^{2} v_{A}^{2}}\right) .
\end{aligned}
$$

Then, starting from Eqs. (4.9), (4.12), and (4.13), we arrive at

$$
\begin{aligned}
& {\left[\left(1+\frac{\gamma^{2}}{k_{z}^{2} v_{A}^{2}}\right)^{2}+\frac{4 \Omega_{2}^{2} \gamma^{2}}{k_{z}^{4} v_{A}^{4}}\right]^{1 / 2}+} \\
& +\left[\left(1+\frac{\gamma^{2}}{k_{z}^{2} v_{A}^{2}}\right)^{2}+\frac{4 \Omega_{1}^{2} \gamma^{2}}{k_{z}^{4} v_{A}^{4}}\right]^{1 / 2}+\frac{r_{0}\left(\Omega_{2}^{2}-\Omega_{1}^{2}\right)}{\left|k_{z}\right| v_{A}^{2}}=0,
\end{aligned}
$$

where $\gamma^{2}=-\omega^{2}$.

\section{SLOW AXISYMMETRIC MODES IN THE SIMPLEST ASTROPHYSICAL HIGH- $\beta$ PLASMA}

\subsection{General properties of nonlocal modes}

One can see that, since the two first terms on the right-hand side of Eq. (4.18) are positive, this equation can be satisfied only if

$$
\Omega_{1}^{2}>\Omega_{2}^{2}
$$

This condition shows that the instability is possible only for a decreasing profile of the rotation frequency, i.e., for $d \Omega^{2} / d \ln r<0$, which agrees with the results of [1-3].

\subsection{The case of a magnetized plasma, $\gamma \ll k_{z} v_{A}$}

The perturbations described by (4.18) are marginally stable $(\gamma=0)$ for $k_{z}=k_{z 0}$ with

$$
\left|k_{z 0}\right|=r_{0}\left(\Omega_{1}^{2}-\Omega_{2}^{2}\right) / 2 v_{A}^{2} .
$$

Turning to (B.5), one can see that, instead of (5.2), the local theory yields Eq. (B.6). It can be seen that (B.6) is in a qualitative agreement with (5.2).
Near the instability boundary, at $\gamma^{2} \ll k_{z}^{2} v_{A}^{2}$, Eq. (4.18) reduces to

$$
\gamma^{2}=\frac{1}{2}\left|k_{z 0}\right| r_{0} \frac{\Omega_{1}^{2}-\Omega_{2}^{2}}{1+\left(\Omega_{1}^{2}+\Omega_{2}^{2}\right) /\left(k_{z 0} v_{A}\right)^{2}}\left(1-\frac{\left|k_{z}\right|}{\left|k_{z 0}\right|}\right) .
$$

At the same time, the expression for the squared growth rate near the instability boundary following from the local dispersion relation (B.5) is given by (B.8). One can see that there is a qualitative agreement between (B.8) and (5.3).

The instability condition following from (5.3) takes the form

$$
\left|k_{z}\right|<\left|k_{z 0}\right|
$$

or, in the explicit form,

$$
\Omega_{1}^{2}-\Omega_{2}^{2}>2 v_{A}^{2}\left|k_{z}\right| / r_{0} .
$$

One can see that, as for the local perturbations [1], sufficiently small wave number is necessary for the nonlocal instability. This agrees with the general results of $[2,3]$.

The above relations describe the nonlocal axisymmetric magnetorotational instability (MRI) in the simplest astrophysical high- $\beta$ plasma, which is physically the same as the local MRI [1].

\subsection{The case of nonmagnetized plasma}

It follows from Eq. (4.18) for $v_{A}^{2} \rightarrow 0$ that

$$
\gamma\left[\left(\gamma^{2}+4 \Omega_{2}^{2}\right)^{1 / 2}+\left(\gamma^{2}+4 \Omega_{1}^{2}\right)^{1 / 2}\right]=r_{0}\left|k_{z}\right|\left(\Omega_{1}^{2}-\Omega_{2}^{2}\right)
$$

For $\gamma \ll\left(\Omega_{2}, \Omega_{1}\right)$ one hence finds

$$
\gamma=\frac{r_{0}\left|k_{z}\right|}{2\left(\left|\Omega_{2}\right|+\left|\Omega_{1}\right|\right)}\left(\Omega_{1}^{2}-\Omega_{2}^{2}\right) .
$$

Since we assumed $r_{0}\left|k_{z}\right| \gg 1$, this equation should be considered for $\left|\Omega_{1}-\Omega_{2}\right| \ll \Omega_{1}$, when it turns into

$$
\gamma=r_{0}\left|k_{z}\right|\left(\Omega_{1}-\Omega_{2}\right) / 4 .
$$

In the opposite case $\gamma \gg\left(\Omega_{2}, \Omega_{1}\right)$ Eq. (5.6) yields

$$
\gamma^{2}=r_{0}\left|k_{z}\right|\left(\Omega_{1}^{2}-\Omega_{2}^{2}\right) / 2
$$

For $\gamma \simeq\left(\Omega_{2}, \Omega_{1}\right)$ one can take $\Omega_{2}=\Omega_{1}=\bar{\Omega}$ on the left-hand side of Eq. (5.6), where $\bar{\Omega}=\left(\Omega_{1}+\Omega_{2}\right) / 2$. Then (5.6) reduces to

$$
\gamma\left(\gamma^{2}+4 \bar{\Omega}^{2}\right)^{1 / 2}=r_{0}\left|k_{z}\right| \bar{\Omega}\left(\Omega_{1}-\Omega_{2}\right)
$$

This equation can be transformed into the bi-quadratic with respect to $\gamma$ with the rule that $\operatorname{Im} \omega>0$ :

$$
\omega^{2}\left(\omega^{2}-4 \bar{\Omega}^{2}\right)=r_{0}^{2} k_{z}^{2} \bar{\Omega}^{2}\left(\Omega_{1}-\Omega_{2}\right)^{2} .
$$

It hence follows that

$$
\begin{gathered}
\omega_{1}=i 2^{1 / 2}|\bar{\Omega}|\left[(1+\Delta)^{1 / 2}-1\right], \\
\omega_{2,3}= \pm 2^{1 / 2}|\bar{\Omega}|\left[(1+\Delta)^{1 / 2}+1\right],
\end{gathered}
$$


where

$$
\Delta=r_{0}^{2} k_{z}^{2}\left(\Omega_{1}-\Omega_{2}\right)^{2} /\left(4 \bar{\Omega}^{2}\right) .
$$

The roots $\omega_{2,3}$ are real, they are not of interest for the stability problem. The root $\omega_{1}$ is generalization of Eqs. (5.8) and (5.9) for arbitrary $\Delta$.

As a whole, the result of this subsection is compatible with the local solution $\gamma^{2}=k_{z}^{2} \kappa^{2} / k^{2}\left(\right.$ for $\left.\kappa^{2}<0\right)$ mentioned in Appendix $B$.

\section{SLOW NONLOCAL AXISYMMETRIC MODES IN A HIGH- $\beta$ PLASMA WITH GRAVITATION FORCE AND PRESSURE GRADIENT}

\subsection{General dispersion relation for low heat conductivity}

For axisymmetric modes, $k_{y}=0, \Delta_{T} \rightarrow$ and $p_{0}^{\prime} \neq 0$, Eqs. (3.2)-(3.4) reduce to

$$
\begin{gathered}
C_{1}=-D_{0} p_{0}^{\prime} /\left(\alpha_{s} v_{A}^{2} \rho_{0}\right), \\
C_{2}=D_{0} / B_{0}^{2} \\
C_{3}=4 \pi \rho_{0} D_{0}\left[( 1 + \frac { \beta } { \alpha _ { s } } ) \left(D_{0}-\frac{d \Omega^{2}}{d \ln r}-\right.\right. \\
\left.\left.-\frac{p_{0}^{\prime}}{r \rho_{0}} \frac{d \ln \rho_{0}}{d \ln r}-\frac{4 \Omega^{2} \omega^{2}}{D_{0}}\right)+\frac{D_{0}}{\alpha_{S} v_{A}^{2} \omega^{2}} \frac{p_{0}^{\prime 2}}{\rho_{0}^{2}}\right] .
\end{gathered}
$$

Here $\beta=c_{s}^{2} / v_{A}^{2}, c_{s}^{2}=\Gamma p_{0} / \rho_{0}$ is the squared sound velocity, $\alpha_{s}=1-k_{z}^{2} c_{s}^{2} / \omega^{2}$. Then Eq. (4.12) yields

$$
I=\left.4 \pi r_{0}\left[\rho_{0}\left(\Omega^{2}-\frac{p_{0}^{\prime}}{r_{0} \rho_{0} \alpha_{s}}\right)\right]\right|_{1} ^{2} .
$$

Turning to (4.13) and using (6.1)-(6.3), we find

$$
(G H)_{2,1}^{1 / 2}=4 \pi r_{0} v_{A}^{2}\left(1+\beta / \alpha_{s}\right)^{1 / 2}\left[-\left(\bar{D}-4 \Omega^{2} \omega^{2} / D_{0}\right)\right]_{2,1}^{1 / 2},
$$

where

$$
\bar{D}=D_{0}-k_{z}^{2} p_{0}^{\prime 2} /\left(\omega^{2} \alpha_{s} \rho_{0}^{2}\right)-p_{0}^{\prime}\left(\ln p_{0}\right)^{\prime} / \rho_{0} .
$$

As a result, (4.9) reduces to

$$
\begin{aligned}
& \left(1+\beta / \alpha_{s}\right)^{1 / 2}\left\{\left[-\left(\bar{D}-4 \Omega^{2} \omega^{2} / D_{0}\right)\right]_{2}^{1 / 2}+\right. \\
& \left.\left[-\left(\bar{D}-4 \Omega^{2} \omega^{2} / D_{0}\right)\right]_{1}^{1 / 2}\right\}+\left.\frac{r_{0}}{v_{A}}\left(\Omega^{2}-\frac{p_{0}^{\prime}}{r_{0} \rho_{0} \alpha_{s}}\right)\right|_{1} ^{2}=0 .
\end{aligned}
$$

\subsection{Slow modes in high- $\beta$ plasma for low heat conductivity}

It follows from (6.7) for $\beta \gg 1$ and $\alpha_{s} \rightarrow-c_{s}^{2} k_{z}^{2} / \omega^{2}$ that

$$
\begin{aligned}
& \left(1+\frac{\gamma^{2}}{k_{z}^{2} v_{A}^{2}}\right)^{1 / 2}\left[\gamma^{2}+k_{z}^{2} v_{A}^{2}-\frac{p_{0}^{\prime 2}}{c_{S}^{2} \rho_{0}^{2}}+\frac{p_{0}^{\prime}}{\rho_{0}}\left(\ln p_{0}\right)^{\prime}\right]^{1 / 2}+ \\
& +\frac{r_{0}}{2 v_{A}}\left(\Omega_{2}^{2}-\Omega_{1}^{2}\right)=0 .
\end{aligned}
$$

It is allowed here that for $\beta \gg 1$ the jump in the pressure gradient is small as $1 / \beta$.

Taking here $\gamma \rightarrow 0$, one arrives at the instability boundary [cf. (B.15)]

$$
\left[k_{z}^{2} v_{A}^{2}-\frac{p_{0}^{\prime}}{\Gamma \rho_{0}} \frac{d}{d r} \ln \left(\frac{p_{0}^{\prime}}{\rho_{0}^{\Gamma}}\right)\right]^{1 / 2}+\frac{r_{0}}{2 v_{A}}\left(\Omega_{2}^{2}-\Omega_{1}^{2}\right)=0 .
$$

Turning to (B.11), Eq. (6.9) is represented as

$$
\left(k_{z}^{2} v_{A}^{2}+N_{\mathrm{BV}}^{2}\right)^{1 / 2}+\frac{r_{0}}{2 v_{A}}\left(\Omega_{2}^{2}-\Omega_{1}^{2}\right)=0,
$$

where $N_{\mathrm{BV}}^{2}$ is defined by (B.11).

For $N_{\mathrm{BV}}^{2}>0$ this dispersion relation describes convective suppression of the nonlocal MRI. On the other hand, for

$$
-k_{z}^{2} v_{A}^{2}<N_{\mathrm{BV}}^{2}<0
$$

Eq. (6.10) describes the convective enhancement of this instability. In the case

$$
N_{\mathrm{BV}}^{2}<-k_{z}^{2} v_{A}^{2}
$$

the nonlocal MRI disappears and the nonlocal CI does not occur.

\subsection{Slow modes in high- $\beta$ plasma for high heat conductivity}

According to [15], transition from the case of low to high heat conductivity is done by

$$
\Gamma \rightarrow 1
$$

in the final formulas. Then one arrives at the instability condition (6.10) with the substitution

$$
N_{\mathrm{BV}}^{2} \rightarrow N_{\mathrm{BV}}^{2 \infty}
$$

where [cf. (B11)]

$$
N_{\mathrm{BV}}^{2 \infty}=-\frac{p_{0}^{\prime}}{\rho_{0}} \ln \left(p_{0} / \rho_{0}\right)^{\prime} .
$$

\section{SLOW NONAXISYMMETRIC MODES IN THE SIMPLEST ASTROPHYSICAL HIGH- $\beta$ PLASMA}

In the case of uniform plasma pressure, $p_{0}^{\prime}=0$, one has by means of (4.12) and (4.13)

$$
I=r_{0}\left\{\Omega_{2}^{2}-\Omega_{1}^{2}-2 \frac{m}{r_{0}^{2}}\left[\frac{\Omega \widetilde{\omega}}{D_{\theta}}\left(v_{A}^{2}+\frac{c_{s}^{2}}{\alpha_{s}}\right)\right]_{1}^{2}\right\}
$$

$$
(G H)_{2,1}^{1 / 2}=\left\{\left(v_{A}^{2}+\frac{c_{s}^{2}}{\alpha_{s}}\right) \frac{\widetilde{\omega}^{2}-k_{z}^{2} v_{A}^{2}}{D_{\theta}}\left[k_{z}^{2} v_{A}^{2}-\widetilde{\omega}^{2}\left(1-\frac{4 \Omega^{2}}{D_{\theta}}\right)\right]\right\}_{2,1}^{1 / 2},
$$

where

$$
D_{\theta}=\widetilde{\omega}^{2}-\left(k_{z}^{2}+k_{y}^{2}\right) v_{A}^{2}-k_{y}^{2} c_{s}^{2} / \alpha_{s}
$$


Then one should substitute (7.1) and (7.2) into the nonlocal dispersion relation (4.9).

For the slow modes in the case $\beta \rightarrow \infty$ Eqs. (7.1) and (7.2) yield [5]

$$
\begin{array}{r}
I=\frac{r_{0}}{k_{y}^{2}+k_{z}^{2}}\left[\left(\Omega_{2}^{2}-\Omega_{1}^{2}\right)\left(k_{z}^{2}-k_{y}^{2}\right)+\frac{2 m}{r_{0}^{2}} \omega\left(\Omega_{2}-\Omega_{1}\right)\right], \\
(G H)_{1,2}^{1 / 2}=\frac{1}{\left(k_{z}^{2}+k_{y}^{2}\right)^{1 / 2}}\left[\left(k_{z}^{2} v_{A}^{2}-\widetilde{\omega}^{2}\right)^{2}-4 \Omega^{2} \widetilde{\omega}^{2} \frac{k_{z}^{2}}{k_{z}^{2}+k_{y}^{2}}\right]_{1,2}^{1 / 2} .
\end{array}
$$

We restrict ourselves to the case of weak rotation frequency jump, so that

$$
\Omega_{1}^{2}-\Omega_{2}^{2} \ll \Omega_{1}^{2}
$$

Then one can introduce the axilliary Doppler-shifted oscilla-

tion frequency

$$
\widehat{\omega}=\omega-m \bar{\Omega},
$$

where

$$
\bar{\Omega}=\left(\Omega_{1}+\Omega_{2}\right) / 2 .
$$

At the same time, in (7.5) we take for simplicity

$$
\widehat{\omega} \gg m\left|\Omega_{1}-\Omega_{2}\right| / 2 \text {. }
$$

We assume $\widehat{\omega}$ a small parameter and find it by the method of successive approximations. In terms of $\widehat{\omega}$ Eqs. (7.4) and (7.5) transit to

$$
I=\frac{r_{0}}{k_{y}^{2}+k_{z}^{2}}\left[k_{z}^{2}\left(\Omega_{2}^{2}-\Omega_{1}^{2}\right)+\frac{2 m}{r_{0}^{2}} \widehat{\omega}\left(\Omega_{2}-\Omega_{1}\right)\right],
$$

$$
(G H)_{2}^{1 / 2}=(G H)_{1}^{1 / 2}=\frac{k_{z}^{2} v_{A}^{2}}{\left(k_{z}+k_{y}^{2}\right)^{1 / 2}}\left\{1-\frac{\widehat{\omega}^{2}}{k_{z}^{2} v_{A}^{2}}\left[1+\frac{2 \bar{\Omega}^{2}}{v_{A}^{2}\left(k_{z}^{2}+k_{y}^{2}\right)}\right]\right\}
$$

Using (4.9), (7.10), and (7.11), we obtain the dispersion relation [5]

$$
\left[1+\frac{2 \bar{\Omega}^{2}}{\left(k_{z}^{2}+k_{y}^{2}\right) v_{A}^{2}}\right] \widehat{\omega}^{2}+f=0,
$$

where

$$
f=k_{z}^{2}\left[\frac{r_{0}\left(\Omega_{1}^{2}-\Omega_{2}^{2}\right)}{2\left(k_{z}^{2}+k_{y}^{2}\right)^{1 / 2}}-v_{A}^{2}\right] .
$$

Hence we find that the perturbations are unstable for

$$
f>0 \text {. }
$$

For $k_{y}=0$ this instability condition reduces to (5.5). Comparing (7.14) with (5.5), one can see that the driving of the nonlocal nonaxisymmetric modes is weakened as $\left|k_{z}\right| /\left(k_{z}^{2}+k_{y}^{2}\right)^{1 / 2}$ compared with that of the axisymmetric modes. This conclusion is compatible with that for the local nonaxisymmetric modes discussed in Appendix C.

\section{DISCUSSION}

The first step to the equations describing the surface-wave instabilities is derivation of the MHD canonical mode equations. According to explanations of Sec. 1, our task is to derive expressions for the primary canonical parameters $D, C_{1}, \bar{C}_{1}, C_{2}$, and $C_{3}$. In general form, these parameters are given by (2.30)(2.32), (1.8) and (2.36). In the quasi-incompressible approximation, instead of these equations, one has (3.1)-(3.4).
Using our technique, we have analyzed applicability of the Boussinesq approximation for studying the slow modes in high- $\beta$ plasma. Then we have found that this approximation leads to a non-self-adjoint equation (1.19). By this reason, it is, in general, unacceptable for such analysis. This drawback of the Boussinesq approximation can be eliminated by an artificial omitting of the term responsible for the non-selfadjointness. This has been done by Balbus in Ref. 6 .

We have considered the surface-wave perturbations in the approximation of step-like equilibrium parameters. Then we have derived general nonlocal dispersion relation (4.9) with its local and nonlocal parts given by Eqs. (4.10) and (4.11), respectively. Reduced version of this dispersion relation for the case of slow axisymmetric modes in the simplest astrophysical plasma for low heat conductivity is given by Eq. (4.18). Analysis of Eq. (4.18) has lead to results presented in Sec. 5. Then we have shown that the nonlocal axisymmetric MRI, as the local one, is possible only for decreasing rotation frequency profile, see Eq. (5.1). The instability boundary is given by Eq. (5.2) showing that, as in the local case, only the perturbations with sufficiently small $k_{z}$ can be unstable. The growth rate of the nonlocal MRI near its instability boundary is given by Eq. (5.3). The results of nonlocal and local theories show that the both varieties of the axisymmetric MRI must be similar.

With equilibrium pressure gradient, $p_{0}^{\prime} \neq 0$, in addition to the surface-wave MRI, the surface-wave convective instability can be revealed, see Sec. 6. In this case the situation with the surface-wave instabilities is similar to that mentioned in Appendix B for the local instabilities.

A more complicated picture is revealed for the nonlocal nonaxisymmetric MRI because of the radial dependence of the 
Doppler-shifted oscillation frequency $\widetilde{\omega}$. Dispersion relation of nonlocal nonaxisymmetric MRI is given by Eq. (4.9) with complementing Eqs. (7.4) and (7.5). Its instability criterion is given by Eq. (7.14). According to these equations, driving the nonlocal nonaxisymmetric MRI is hampered compared with that of the axisymmetric one.

Numerical calculations of nonlocal nonaxisymemtric MRI have been done in [17-21]. The analytical results presented here will be useful for understanding the results of computations and for further studies on both the nonaxisymemtric and axisymemtric MRIs.

\section{Acknowledgments}

We are grateful to A. I. Smolyakov, I. V. Khalzov and S. V. Vladimirov for valuable discussions. This work was supported in parts by the Russian Foundation of Basic Researches (RFBR), grant No. 06-02-16767, the Russian Program of Support of Leading Scientific Schools, grant \# 9878.2006.2, the
National Council of Science and Technology Development (CNPq), Brazil, the International Science and Technology Center (ISTC), grant \# G-1217, and the Science and Technology Center in Ukraine, grant \# 3473.

\section{Appendix A General results of the theory of local instabilities for low heat conductivity}

In the case of local modes one takes the function $\widetilde{B}_{r}$ in the form

$$
\widetilde{B}_{r}=\bar{B}_{r}(r) \exp \left(i k_{r} r\right)
$$

where $\bar{B}_{r}(r)$ is a slowly varying amplitude. Then (4.4) leads to

$$
-k_{r}^{2} D^{2} / C_{2}+\Lambda=0
$$

which is the local dispersion relation. The explicit form of this dispersion relation for low heat conductivity is
Here

$$
\begin{aligned}
D_{0} & -k_{\perp}^{2} v_{A}^{2}\left(1+\beta / \alpha_{s}\right)-\left[\frac{d \Omega^{2}}{d \ln r}-\frac{1}{\alpha_{s}} \frac{d}{d \ln r}\left(\frac{p_{0}^{\prime}}{r \rho_{0}}\right)\right]-\frac{p_{0}^{\prime}}{r \rho_{0} D_{0}} E_{\rho}+ \\
& +\left(\frac{D_{y}}{\widetilde{\omega}^{2}}-\frac{\beta}{\alpha_{s}}\right) \frac{p_{0}^{\prime 2}}{\alpha_{s} v_{A}^{2} \rho_{0}^{2}\left(1+\beta / \alpha_{s}\right)}-\frac{4 \widetilde{\omega}^{2} \Omega^{2}}{D_{0}}-\frac{2 m \Omega \widetilde{\omega}}{D_{0}^{2}} E_{\widetilde{\omega}}=0
\end{aligned}
$$

while $\xi^{\mathrm{NA}}$ is defined by

$$
\begin{aligned}
& E_{\rho}=D_{0}-k_{y}^{2} v_{A}^{2}\left(1+\frac{\beta}{\alpha_{s}}\right) \frac{d \ln \rho_{0}}{d \ln r}-\frac{D_{0}\left(1+\beta / \alpha_{s}\right)}{\alpha_{s}} \xi^{p}, \\
& E_{\widetilde{\omega}}=\left(\frac{D_{y}}{D_{0}}+\frac{\beta}{\alpha_{s}}\right) \frac{p_{0}^{\prime}}{r \rho_{0}}-\frac{D_{0} v_{A}^{2}}{r^{2}}\left(1+\frac{\beta}{\alpha_{s}}\right) \xi^{\mathrm{NA}}
\end{aligned}
$$

where

$$
\xi^{p}=\frac{d}{d \ln r} \ln \frac{D_{0} \rho_{0}}{\alpha_{s} D_{y}}
$$
form
The superscript " $p$ " means a quantity dependent on the pressure gradient, while the superscript "NA" means "nonaxisymmetric". Dispersion relation (A.3) for $p_{0}^{\prime}=0$ is represented in the

$$
D_{0}\left[D_{0}-k_{\perp}^{2} v_{A}^{2}\left(1+\frac{\beta}{\alpha_{s}}\right)-\frac{d \Omega^{2}}{d \ln r}\right]-4 \Omega^{2} \widetilde{\omega}^{2}+\frac{2 \Omega \widetilde{\omega}}{m} k_{y}^{2} v_{A}^{2} \xi^{\mathrm{NA}}=0
$$

Here $k_{\perp}^{2}=k_{r}^{2}+k_{y}^{2}$, and

$$
\xi^{\mathrm{NA}}=\frac{d}{d \ln r} \ln \left\{\frac{\Omega\left(1+\beta / \alpha_{s}\right)}{r^{2}\left[D_{0}-k_{y}^{2} v_{A}^{2}\left(1+\beta / \alpha_{s}\right)\right]}\right\} .
$$

\section{Appendix B Local axisymmetric modes}

\section{B.1. The simplest astrophysical plasma}

For $m=0$ Eq. (A.9) leads to the focal dispersion relation

$$
D_{0}\left[D_{0}-k_{\perp}^{2} v_{A}^{2}\left(1+\frac{\beta}{\alpha_{s}}\right)-\frac{d \Omega^{2}}{d \ln r}\right]-4 \Omega^{2} \widetilde{\omega}^{2}=0 .
$$


For incompressible plasma with $\beta \gg 1$, Eq. (B.1) reduces to

$$
\gamma^{4}+\gamma^{2}\left(2 k_{z}^{2} v_{A}^{2}+\frac{k_{z}^{2}}{k^{2}} \kappa^{2}\right)+k_{z}^{4} v_{A}^{4}\left(1+\frac{1}{k^{2} v_{A}^{2}} \frac{d \Omega^{2}}{d \ln r}\right)=0,
$$

where $k^{2}=k_{r}^{2}+k_{z}^{2}$. The instability boundary is then given by

$$
\left(k^{2}\right)^{\mathrm{loc}}=-\left(d \Omega^{2} / d \ln r\right) / v_{A}^{2} .
$$

The growth rate near the instability boundary is defined by

$$
\left(\gamma^{2}\right)^{\mathrm{loc}}=-k_{z}^{2} v_{A}^{2} \frac{k^{2} v_{A}^{2}+d \Omega^{2} / d \ln r}{2 k^{2} v_{A}^{2}+\kappa^{2}}
$$

Note also that for $v_{A}^{2} \rightarrow 0$ Eq. (B.2) has solution $\gamma^{2}=$ $-k_{z}^{2} \kappa^{2} / k^{2}$. This solution describes the local instability for $\kappa^{2}<0$.

\section{B.2. The local modes in the presence of pressure gradient}

\section{B.2.1. Local dispersion relation near instability boundary}

For small $\omega$ and $k_{y}=0$ one has from (A.3)

$$
\omega^{2} \Delta_{1}^{p}+k_{z}^{2} v_{A}^{2} \Delta^{p}=0,
$$

where

$$
\begin{gathered}
\Delta^{p}=-\left[1+\frac{1}{k^{2} v_{A}^{2}}\left(\frac{d \Omega^{2}}{d \ln r}-\frac{p_{0}^{\prime 2}}{\rho_{0}^{2} c_{s}^{2}}+\frac{p_{0}^{\prime}}{\rho_{0} r} \frac{d \ln \rho_{0}}{d \ln r}\right)\right], \\
\Delta_{1}^{p}=1+\frac{\Omega^{2}}{k^{2} v_{A}^{2}}\left[4+\frac{1}{\beta}\left(\frac{d \ln p_{0}}{d \ln r}-\frac{d \ln \rho_{0}}{d \ln r}\right)\right] .
\end{gathered}
$$

Then the dispersion relation (B.5) has the roots

$$
\omega^{2}=-k_{z}^{2} \Delta^{p} / \Delta_{1}^{p}
$$

One of these roots describes unstable modes for

$$
\Delta^{p}<0 .
$$

Neglecting the term with $p_{0}^{\prime}$ in Eq. (B.6), one has

$$
\Delta^{p}=\Delta^{p V} \equiv 1+\frac{1}{k^{2} v_{A}^{2}} \frac{d \Omega^{2}}{d \ln r} .
$$

Then Eq. (B.9) is the Velikhov instability condition. The term with $p_{0}^{\prime}$ in Eq. (B.6) is responsible for the convective instability, CI. Following [1], one can introduce the Brunt-Väisälä (BV) frequency $N_{B V}$ defined by

$$
N_{B V}^{2}=-\frac{p_{0}^{\prime}}{\Gamma \rho_{0}}\left(\ln \frac{p_{0}}{\rho_{0}^{\Gamma}}\right)^{\prime}
$$

Then Eq. (B.6) is represented as

$$
\Delta^{p}=\Delta^{p V}+\Delta^{p B}
$$

where

$$
\Delta^{p B}=N_{B V}^{2} /\left(k^{2} v_{A}^{2}\right)
$$

The BV effect is destabilizing for

$$
N_{B V}^{2}<0 \text {. }
$$

With Eq. (B.6), the explicit form of the instability condition (B.9) is

$$
k^{2} v_{A}^{2}+\frac{d \Omega^{2}}{d \ln r}-\frac{p_{0}^{\prime}}{\Gamma \rho_{0}} \frac{d}{d r} \ln \left(\frac{p_{0}}{\rho_{0}^{\Gamma}}\right)<0 .
$$

This result coincides with that obtained in Ref. 1.

\section{Appendix C Local nonaxisymmetric modes in the simplest astrophysical plasma}

Taking $\widetilde{\omega}$ to be a small parameter, we reduce (A.10) to

$$
\xi^{\mathrm{NA}}=d \ln \Omega / d \ln r \text {. }
$$

Then (A.9) is transformed to

$D_{0}\left[D_{0}-k_{\perp}^{2} v_{A}^{2}\left(1+\frac{\beta}{\alpha_{s}}\right)-\frac{d \Omega^{2}}{d \ln r}\right]-4 \Omega^{2} \widetilde{\omega}^{2}+\frac{2 \widetilde{\omega}}{m} \frac{d \Omega}{d \ln r} k_{y}^{2} v_{A}^{2}=0$.

For $\beta \rightarrow \infty$ one has from (C.2)

$$
\widetilde{\omega}^{2} \Delta_{1}^{\mathrm{NA}}-\frac{2 \widetilde{\omega}}{m} \frac{d \Omega}{d \ln r} \frac{k_{y}^{2}}{k_{z}^{2}+k_{\perp}^{2}}+k_{z}^{2} v_{A}^{2} \Delta^{\mathrm{NA}}=0,
$$

where

$$
\begin{aligned}
& \Delta^{\mathrm{NA}}=-\left[1+\frac{1}{\left(k_{z}^{2}+k_{\perp}^{2}\right) v_{A}^{2}} \frac{d \Omega^{2}}{d \ln r}\right], \\
& \Delta_{1}^{\mathrm{NA}}=1+\frac{4 \Omega^{2}}{v_{A}^{2}\left(k_{z}^{2}+k_{\perp}^{2}\right)},
\end{aligned}
$$

the superscript "NA" means "nonaxisymmetric".

\section{C.1.1 The approximation $\widetilde{\omega} / m \rightarrow 0$}

Without the term with $\widetilde{\omega} / m \mathrm{Eq}$. (C.3) reduces to

$$
\gamma^{2}=k_{z}^{2} v_{A}^{2} \Delta^{\mathrm{NA}} / \Delta_{1}^{\mathrm{NA}}
$$

Then the instability boundary is given by

$$
\Delta^{\mathrm{NA}}=0 \text {. }
$$

The nonaxisymmetric instability region corresponds to

$$
\Delta^{\mathrm{NA}}>0 \text {. }
$$

At the same time, starting with (C.3), one can obtain that for the case of axisymmetric modes, $k_{y}=0$, Eq. (C.6) is substituted by

$$
\gamma^{2}=k_{z}^{2} v_{A}^{2} \Delta / \Delta_{1}
$$

where

$$
\Delta-\left[1+\frac{1}{\left(k_{z}^{2}+k_{r}^{2}\right) v_{A}^{2}} \frac{d \Omega^{2}}{d \ln r}\right]
$$

$$
\Delta_{\mp} 1+4 \Omega^{2} /\left[\left(k_{z}^{2}+k_{r}^{2}\right) v_{A}^{2}\right] .
$$

Comparing (C.6) with (C.10), we conclude that the nonaxisymmetric perturbations are less dangerous than the axisymmetric ones. 


\section{References}

[1] S. A. Balbus and J. F. Hawley, Astrophys. J. 376, 214 (1991).

[2] E. P. Velikhov, Sov. Phys. JETP 36, 995 (1959).

[3] S. Chandrasekhar, Proc. Natl. Acad. Sci. USA 46, 253 (1960).

[4] H. Ji, P. Kronberg, S. C. Prager, and D. A. Uzdensky, Phys. Plasmas 15, 058302 (2008).

[5] A. B. Mikhailovskii, J. G. Lominadze, R. M. O. Galvão, A. P. Churikov, O. A. Kharshiladze, N. N. Erokhin, and C. H. S. Amador, Phys. Plasmas 15, 052109 (2008).

[6] S. A. Balbus, Astrophys. J. 562, 909 (2001).

[7] E. Quataert, Astrophys. J. 673, 758 (2008).

[8] A. Socrates, I. J. Parrish, and J. M. Stone, Astrophys. J. 675, 357 (2008).

[9] A. B. Mikhailovskii, J. G. Lominadze, A. P. Churikov, N. N. Erokhin, V. D. Pustovitov, and S. V. Konovalov, Axisymmetric magnetorotational instability in ideal and viscous plasma, Plasma Phys. Rep. 34, 837 (2008).

[10] A. B. Mikhailovskii, J. G. Lominadze, R. M. O. Galvão, A. P. Churikov, N. N. Erokhin, A. I. Smolyakov, and V. S. Tsypin, Phys. Plasmas 15052103 (2008).

[11] E. Frieman and N. Rotenberg, Rev. Mod. Phys. 32, 898
(1960).

[12] E. Hameiri, J. Math. Phys. 22, 2080 (1981).

[13] A. Bondeson, R. Iacono and A. Bhattacharjee, Phys. Fluids 30, 2167 (1987).

[14] R. Keppens, F. Casse and J. P. Goedbloed, Astrophys. J. 569, L121 (2002).

[15] A. B. Mikhailovskii, J. G. Lominadze, A. I. Smolyakov, A. P. Churikov, V. D. Pustovitov, and O. A. Kharshiladze, Heatconductivity-induced instabilities in a rotating plasma, Bul. Georg. Nat. Acad. Sci., to be published (2009).

[16] A. B. Mikhailovskii, A. I. Smolyakov, J. G. Lominadze, A. P. Churikov, V. D. Pustovitov, and O. A. Kharshiladze, Effects of finite heat conductivity on instabilities in a rotating plasma, Plasma Phys. Rep. to be published (2009).

[17] I. V. Khal'zov, V. I. Ilgisonis, A. I. Smolyakov, and E. P. Velikhov, Phys. Fluids 18, 124107 (2006).

[18] L. L. Kitchatinov and G. Rudiger, Mon. Not. Roy, Astron. Soc. 286, 757 (1997).

[19] G. Rudiger, L. Primavera, R. Arlt, and D. Elsner, Mon. Not. Roy Astron. Soc. 306, 913 (1999).

[20] D. A. Shalybkov, G. Rudiger, and M. Shultz, Astron. Astrophys. 395, 339 (2002).

[21] G. Rudiger, M. Shultz, and D. Shalybkov, Phys. Rev. E 67, 046312 (2003). 